\title{
Aprendizaje basado en la resolución de problemas: una experiencia práctica
}

\author{
E. González-López a , I. García-Lázaro b, A. Blanco-Alfonso `, A. Otero-Puime d
}

El aprendizaje basado en la resolución de problemas incorpora herramientas metodológicas capaces de facilitar la consecución de los objetivos propuestos para la formación de los futuros médicos dentro del marco de la docencia universitaria en el Espacio Europeo de Educación Superior. Promueve una formación más activa, flexible y práctica, que concede mayor protagonismo al trabajo personal tutorizado (aprendizaje autodirigido), en detrimento de las clásicas clases teóricas, eminentemente expositivas, en las que el papel del estudiante es, en general, más pasivo. La Unidad de Medicina de Familia de la Universidad Autónoma de Madrid incorporó el aprendizaje basado en la resolución de problemas en el desarrollo de la asignatura optativa 'Atención Primaria y Medicina de Familia', ofertada como optativa a los alumnos de segundo ciclo de licenciatura (cursos 4. ${ }^{\circ}$ a 6..$^{\circ}$ ) desde el curso 2005-2006. Intentamos con ella promover la formación de médicos capaces de aprender y mantener su competencia durante toda su vida profesional, no sólo en lo referido a la adquisición/integración de conocimientos científicos suficientes, sino también en cuanto al desarrollo de las habilidades necesarias para su adecuada aplicación práctica considerando a cada paciente de modo integral como realidad biopsicosocial, en un contexto sanitario definido, sin olvidar los aspectos bioéticos implícitos al quehacer del médico (respeto hacia el paciente y compromiso social). Revisamos en este artículo el diseño práctico de la asignatura.

Palabras clave. Aprendizaje autodirigido. Aprendizaje basado en la resolución de problemas. Aprendizaje basado en problemas. Autoaprendizaje dirigido. Resolución de problemas.

\section{Problem-based-solving learning: a practical experience}

Learning based on problem-solving incorporates methodological tools that make it easier to fulfil the aims set for the training of future physicians within the framework of university education in the European Higher Education Area. It encourages a more active, flexible and practical training, which grants a leading role to tutor-guided personal work (self-directed learning) rather than the classical theoretical, eminently expository, classes, in which the student generally played a more passive role. The Family Medicine Unit at the Universidad Autónoma de Madrid has included learning based on problem-solving as part of the elective subject 'Primary Care and Family Medicine', which is optional for students in the second cycle of their degree (years 4 to 6), since the academic year 2005-2006. By so doing our aim is to try to promote the training of physicians who are capable of learning and maintaining their competence throughout their entire career. This must not only involve the acquisition/ integration of sufficient scientific knowledge but also the development of the skills needed to be able to apply them in a practical sense by considering each patient in a integral manner as a biopsychosocial reality, within a particular health care context. And we must not forget the bioethical aspects that are implicit in the doctor's work (respect for the patient and social compromise). In this article, we review the practical design of the subject.

Key words. Directed self-learning. Learning based on problem-solving. Problem-based learning. Problem-solving. Self-directed learning.

\section{Introducción}

El modelo clásico de enseñanza de la medicina basado únicamente en la transmisión expositiva de conocimientos por parte del profesor puede no ser el método adecuado para formar médicos capaces de afrontar con éxito el ejercicio profe- a Centro de Salud Universitario Villanueva de la Cañada.

b Centro de Salud Universitario Ciudad de los Periodistas.

c Centro de Salud Universitario Reina Victoria.

d Cátedra UAM Novartis de Medicina de Familia y Atención Primaria. Facultad de Medicina. Universidad Autónoma de Madrid. Madrid, España

\section{Correspondencia}

Dr. Esteban González López. Centro de Salud Universitario Villanueva de la Cañada. Avda. Valle Baztán, 4. E-28692 Villafranca del Castillo (Madrid).

E-mail estebangl@wanadoo.es

Dedicatoria

A todos los profesores $y$ colaboradores docente clínicos de la Unidad Docente de Medicina de Familia de la Facultad de Medicina de la Universidad Autónoma de Madrid y de sus centros de salud universitarios, quienes, curso tras curso y desde hace muchos años, trabajan en algo tan primordial como formar buenos médicos. 
Tabla I. Características generales del ABP.

\begin{tabular}{|c|c|}
\hline $\begin{array}{l}\text { Comienza con el problema o elemento } \\
\text { desencadenante que dirige el aprendizaje }\end{array}$ & $\begin{array}{l}\text { Problema seleccionado por el equipo docente } \\
\text { para cubrir las necesidades formativas }\end{array}$ \\
\hline $\begin{array}{l}\text { Centrado en el alumno y } \\
\text { sus necesidades de información }\end{array}$ & $\begin{array}{l}\text { No hay que conocer toda la teoría para poder afrontar } \\
\text { básicamente un problema clínico } \\
\text { El alumno tiene conocimientos básicos de los que hay que partir } \\
\text { Ameno y humanamente más satisfactorio }\end{array}$ \\
\hline Activo: el alumno es protagonista de su aprendizaje & $\begin{array}{l}\text { El alumno busca información, la evalúa y decide } \\
\text { sobre la aplicación o utilización de la misma }\end{array}$ \\
\hline Se trabaja en pequeños grupos & $\begin{array}{l}\text { Donde todos aprenden de todos } \\
\text { Estimula la responsabilidad frente al grupo } \\
\text { Facilita las habilidades de comunicación } \\
\text { Fomenta el trabajo en equipo y el respeto a los demás }\end{array}$ \\
\hline $\begin{array}{l}\text { El profesor abandona el rol tradicional } \\
\text { de impartir clases sólo teóricas para pasar } \\
\text { a ser un tutor-facilitador, capaz de crear } \\
\text { entornos donde el aprendizaje sea inevitable }\end{array}$ & $\begin{array}{l}\text { El tutor no necesariamente es experto en el problema } \\
\text { Anima a todos a participar } \\
\text { Previene desviaciones en el aprendizaje } \\
\text { Asegura que el grupo alcance los objetivos de aprendizaje } \\
\text { Comprueba la comprensión } \\
\text { Valora la tarea y participación de cada alumno }\end{array}$ \\
\hline
\end{tabular}

sional en la sociedad actual y futura [1]. Y ello por varias razones.

En primer lugar, el objetivo de enseñar 'toda la medicina' en 6 cursos, inviable hace años, hoy lo es en mayor medida: Los avances científicos y tecnológicos se producen y difunden cada vez a mayor velocidad, siendo fundamental la formación en áreas que capaciten al estudiante para identificar, seleccionar y acceder adecuadamente a la información científica actualizada y relevante con el objetivo de optimizar la calidad de su práctica clínica en cualquier ámbito de trabajo. Nuestro objetivo no es ya la formación de 'médicos generales', cuyo ejercicio requiere también un aprendizaje especializado en Medicina de Familia, sino la formación de profesionales polivalentes y flexibles capaces de responsabilizarse de su propia autoformación y de trabajar en entornos cambiantes [2]. De ahí la importancia de 'aprender a aprender' [3].

Por otra parte, la enseñanza tradicional de la medicina se basa en la transmisión de conocimientos con frecuencia demasiado compartimentados por órganos, aparatos y/o sistemas, $\mathrm{y}$ con una nítida separación entre disciplinas básicas y disciplinas clínicas, lo que a menudo im- pide al estudiante captar que en cada paciente, como ser único, coexisten e interactúan varios problemas en diferentes estadios y que deben ser controlados a la vez.

La enseñanza clásica asumía que demostrar que se sabía la patología equivalía a demostrar que se sabía diagnosticar y manejar al enfermo aquejado de ese problema, sin prestar, en general, suficiente atención específica al aprendizaje de actitudes y habilidades que facilitasen la implementación práctica del conocimiento teórico.

En la enseñanza convencional de la medicina, tampoco se ha recibido formación específica en materias transversales, básicas para todas las especialidades clínicas como la entrevista clínica, el razonamiento clínico, la toma de decisiones en entornos de incertidumbre y la bioética, sin olvidar la importancia que tienen para la identificación y resolución de los problemas de salud, tanto la consideración de los aspectos humanos y sociales del paciente dentro de su particular contexto sociofamiliar y laboral, como los derivados de la propia estructura de los servicios sanitarios y del entorno de trabajo del médico, incluyendo la relación entre niveles asistenciales y el trabajo en equipos multidisciplinares. 
Tabla II. Ventajas del ABP.

- Permite mantener la competencia

- Prepara para el aprendizaje activo a lo largo de la vida profesional

- Aumenta la motivación para aprender

- Permite identificar conocimientos de los que ya se dispone

- Permite el trabajo en equipo (colaboración y coordinación de esfuerzos)

Con el objetivo de mejorar la formación de los estudiantes de Medicina y facilitar el 'aprender a aprender', surgió hace 40 años la iniciativa de la Universidad McMaster [4] (Canadá) denominada 'Aprendizaje Basado en Problemas' (ABP, o Problem-Based Learning), método de aprendizaje basado en el principio de usar problemas como punto de partida para la adquisición e integración de los nuevos conocimientos [5]. Este modelo fue aplicado posteriormente en la enseñanza de profesiones no médicas [6,7]. Las tablas I, II y III recogen las características generales, ventajas e inconvenientes del $\mathrm{ABP}$ frente a la enseñanza clásica.

La nueva formulación de la educación médica propugnada por McMaster no separaba las ciencias básicas de las ciencias clínicas y abandonaba parcialmente las clases teóricas, que se daban después de la presentación del problema. Se intentaba formar unos médicos que tuvieran habilidades para resolver problemas en la práctica clínica real y fueran capaces de analizar críticamente la información recibida, reteniendo e integrando a adecuada. El crecimiento explosivo de la información médica y la existencia de nuevas tecnologías capaces de facilitar el acceso a dicha información hace todavía más necesario este planteamiento.

La primera promoción de médicos de la Universidad McMaster se graduó en 1972 y desde entonces este método se utiliza en muchas facultades de medicina de Canadá, Estados Unidos y Europa. Aunque el modelo original de currículo de McMaster ha sufrido diferentes modificaciones a lo largo de los años, sus ideas principales se mantienen vigentes y han sido origen de muchos cambios en la enseñanza de la medicina [8-10].
Tabla III. Inconvenientes del ABP.

- Requiere el concurso de más profesores

- Requiere más recursos en biblioteca y salas de informática

- Escasa tradición en el autoaprendizaje dirigido por parte de profesores y alumnos

- Ansiedad entre los alumnos si

no se les tutoriza decuadamente

- La evaluación es más compleja

que con un examen teórico habitual

Aunque algunos profesionales formados con esta metodología creen disponer de menos conocimientos (sobre todo en áreas básicas) que los formados con métodos tradicionales, las observaciones realizadas por evaluadores externos no siempre han confirmado esta percepción en el área de los conocimientos, mientras que los graduados con programas que incorporan el ABP, además de encontrar el método más estimulante y humano, parecen estar mejor preparados en otras habilidades necesarias para la práctica clínica: asumir la incertidumbre, considerar aspectos éticos y de comunicación médico-paciente en la actividad asistencial y mantener su competencia profesional a lo largo del tiempo. Es probable que una combinación de ambos métodos proporcione la educación más efectiva a los estudiantes [11-17].

\section{ABP y el Espacio Europeo de Educación Superior}

El ABP, que supuso una ruptura radical con la enseñanza tradicional, incorpora herramientas metodológicas capaces de facilitar la consecución de los objetivos propuestos para la formación de los futuros médicos dentro del marco de la docencia universitaria en el Espacio Europeo de Educación Superior (EEES) [18-20]. La formación de los futuros graduados médicos ha de ser activa, flexible, práctica, prestando mayor protagonismo al trabajo personal y tutorizado, en detrimento de las clases teóricas expositivas en las que el papel del estudiante es generalmente más pasivo [18-22]. Se trata de conseguir médicos capaces de mantener su competencia y 
aprender durante toda su vida profesional (life long learning).

\section{ABP y medicina basada en la evidencia (MBE)}

La MBE, definida como 'el uso consciente, explícito y juicioso de las mejores pruebas disponibles en la toma de decisiones sobre la atención integral de cada paciente' [23], es una herramienta adecuada para la resolución de dudas o problemas clínicos que puedan tener médicos y alumnos. El conocimiento adecuado de los recursos y metodología de la MBE es un medio para el mantenimiento continuo de la competencia.

Una de las fases del ABP es la búsqueda de información adecuada para responder a las carencias que presenta el estudiante. En una primera fase las preguntas suelen ser muy generales, del tipo: ¿qué tiene el paciente?, ¿por qué?, ¿cómo?, y sus respuestas suelen estar disponibles en los medios más accesibles para el estudiante (textos, apuntes, consulta a expertos). Posteriormente, las dudas se van modulando y haciéndose más precisas y concretas, como lo es el propio contexto y condición del paciente, apareciendo las preguntas del tipo: ¿qué prueba diagnóstica es la más adecuada y rentable?, ¿qué tratamiento es el más oportuno para este tipo de paciente?, ¿qué seguimiento debemos programar en este caso? Para responder estas dudas puede ser necesario realizar una lectura crítica de la literatura biomédica con diferentes niveles de profundización [24], recurriendo a las guías de práctica clínica, a las revisiones sistemáticas, a los metanálisis publicados y llegando hasta los artículos originales, si ello fuese necesario. De este modo incorporamos la MBE al ABP.

\section{ABP y aprendizaje basado en la resolución de problemas (ABRP)}

En el ABP, es el problema el que dirige las necesidades de aprendizaje del estudiante, que se hace consciente de sus carencias y de la necesidad de aprender. El objetivo es que el proceso de aprendizaje lleve al resultado indicado por los objetivos de aprendizaje del programa.
El ABRP es una extensión del ABP que incluye una intervención por parte del estudiante para resolver los problemas planteados en el caso-problema. El alumno aprende a partir de la resolución organizada de problemas clínicos concretos previamente seleccionados por el equipo docente y adquiere la competencia para resolver un problema similar en otra ocasión.

El ABRP es una estrategia formativa que también ayuda a mantener la competencia del posgrado, ya que es motivador formarse para resolver los problemas y las dudas que surgen de la práctica clínica diaria y muy razonable que ellas dirijan, en parte, el aprendizaje y estudio [25]. Del mismo modo, el ABRP es también una estrategia de aprendizaje muy útil para la formación de los médicos residentes: se parte de médicos especialistas en formación y con amplios conocimientos generales que deben identificar lo que saben y seleccionar lo que han de aprender para mejorar su competencia, tanto para el momento actual como para el futuro [26] .

\section{La experiencia en la Facultad de Medicina de la Universidad Autónoma de Madrid}

La Unidad de Medicina de Familia y Atención Primaria, dependiente del Departamento de Medicina de la UAM, imparte, entre otras actividades docentes, una asignatura optativa denominada Medicina de Familia y Atención Primaria (seis créditos) para cuyo desarrollo, y desde el curso 2005-2006, incorporamos la metodología del ABRP dirigido a grupos pequeños.

Los pilares básicos de la asignatura pueden resumirse como sigue:

- El conocimiento se construye sobre la base de problemas reales.

- El alumno adquiere el papel de protagonista y se hace responsable de su propio proceso de aprendizaje.

- El docente deja de ser un mero transmisor de conocimientos y pasa a ser un facilitador del proceso de aprendizaje.

La asignatura se ha diseñado para alumnos del segundo ciclo de la licenciatura de Medicina (cursos $4 .^{\circ}$ y, especialmente, $5 .^{\circ}$ y $6 .^{\circ}$ de carrera) con formación clínica previa. 
Desde el punto de vista formativo, ofrece al estudiante la oportunidad de afianzar e integrar, en un contexto práctico, los conocimientos, actitudes y habilidades adquiridas durante los años previos de carrera, ya que el ámbito de la atención primaria exige al médico el manejo cotidiano de múltiples y variados problemas que competen a todos y cada uno de los campos del saber médico. El marco asistencial permite al estudiante percibir fácilmente la importancia de aspectos de la práctica diaria del médico, como la atención longitudinal a lo largo de la vida del paciente, la atención integrada de todos sus problemas de salud y la atención integral del paciente como ser humano enfermo dentro de un contexto social, familiar y laboral determinados. La asignatura facilita la reflexión y el aprendizaje sobre otras competencias básicas necesarias para la práctica clínica, como el razonamiento clínico, la toma de decisiones en contextos de incertidumbre, la comunicación médico-paciente, el manejo de la información, los conflictos bioéticos y las responsabilidades legales del médico, así como sobre la importancia que tiene la integración-coordinación del trabajo entre diferentes profesionales y entre niveles asistenciales distintos para mejorar la calidad de nuestra práctica clínica.

Estructuralmente, la asignatura incluye una sesión inicial conjunta, una estancia-rotación individual en una consulta de medicina general $\mathrm{y}$ varias sesiones tutorizadas dirigidas a un grupo pequeño de entre cuatro y seis estudiantes.

Durante la sesión inicial de introducción general revisamos con los alumnos los objetivos de esta (para nosotros) novedosa asignatura, así como los aspectos metodológicos prácticos necesarios para su desarrollo, facilitamos el material básico necesario y repasamos alguna de las herramientas necesarias para su correcta implementación, como el razonamiento clínico y la búsqueda bibliográfica.

La estancia-rotación individual en la consulta con los profesores asociados en nuestros centros de salud universitarios, permite el contacto directo del estudiante con un entorno laboral real donde cada día ponemos en práctica la estrategia de trabajo y los conocimientos que pretendemos enseñar.

Las sesiones tutorizadas persiguen que los estudiantes tomen decisiones sobre el manejo clínico práctico del paciente-problema que se les presenta. El caso-problema ha sido elegido específicamente por el grupo de docentes como un ejemplo de la práctica cotidiana del médico de familia en Atención Primaria: sobre el enfermo, y a lo largo de su vida, confluyen determinados problemas clínicos, preventivos, sociales y éticos que los alumnos han de ser capaces de identificar y priorizar antes de formular y planificar sus posibles soluciones, argumentando en cada caso las alternativas propuestas.

Cada sesión tutorizada comienza revisando una parte concreta de la historia clínica del paciente: los estudiantes son los encargados de identificar los problemas del enfermo, discutirlos, priorizarlos y decidir sobre sus dudas y necesidades formativas para resolverlos, así como sobre las fuentes bibliográficas y recursos que van a utilizar para ello. El tutor facilita que el grupo identifique y seleccione los problemas y las necesidades de información de los alumnos y revisa con ellos los posibles medios de información disponibles (revistas médicas, textos, búsqueda de recursos en Internet).

Después de un periodo de trabajo personal, el grupo vuelve a reunirse para revisar las respuestas y las decisiones prácticas propuestas por cada alumno. Se realiza, además, una evaluación crítica tanto de la información recopilada como de las herramientas básicas empleadas en su recuperación, con el objetivo de optimizar la calidad de las búsquedas y las respuestas. Tras ello, se aportan nuevos datos evolutivos de la historia sanitaria del paciente y se repite el ciclo de:

- Identificación-discusión de problemas y necesidades formativas.

- Búsqueda personal de respuestas de calidad.

- Discusión crítica de las posibles soluciones durante la siguiente sesión de grupo.

La última de estas sesiones conjuntas termina en el apartado de identificación de problemas, cuyas posibles soluciones argumentará cada alumno por escrito y de forma individual. Este documento será posteriormente utilizado como parte de la evaluación individual de cada estudiante.

Para facilitar y favorecer el desarrollo de las sesiones tutorizadas y el autoaprendizaje, cada alumno lleva unas fichas de registro y trabajo personal en las que, para cada sesión, anota sus necesidades de formación y áreas de trabajo, así como la información utilizada.

En este proceso de aprendizaje, el profesor actúa como facilitador-supervisor, asegurando que durante las sesiones se discutan los aspectos 
del caso que el equipo docente ha considerado claves. Su principal tarea consiste en crear un entorno en el que el aprendizaje resulte gratificante, estimulante e inevitable.

El trabajo de cada estudiante finaliza con la entrega de un documento que adopta una estructura de portafolio [27] e incluye las fichas de registro y trabajo personal y la resolución del caso clínico presentado. Este es el camino que ha de recorrer cualquier profesional médico para mantenerse actualizado en todos los aspectos de su especialidad y del funcionamiento del sistema sanitario. La calificación de este trabajo junto con la asistencia, participación y aportaciones en las sesiones tutorizadas conforman la nota final de la asignatura.

En los anexos se transcribe una situación clínica práctica (Anexo 1), así como (Anexo 2) los objetivos de aprendizaje seleccionados por el equipo docente en este caso-problema, para cuyo manejo el alumno debe integrar conocimientos adquiridos previamente en otras asignaturas.

\section{Conclusión}

El ABP ha demostrado su utilidad como una estrategia pedagógica que permite generar profesionales motivados y capaces de adquirir, mantener y mejorar su competencia a lo largo de su vida profesional, tanto en el área de los conocimientos como en las de las habilidades y actitudes. El ABRP amplía los objetivos de aprendizaje e incluye la capacitación para la resolución de los problemas en su ámbito profesional.

En cuanto a la ubicación del ABRP en el currículo de Medicina, este método puede ser un complemento de las clases teóricas y prácticas, ya que facilita que el alumno se ejercite en la integración de todo lo aprendido previamente y se haga consciente de lo que necesita saber para la resolución de los problemas concretos de un paciente. Se podría decir que 'pone rostro humano a los apuntes'.

\section{Bibliografía}

1. Cardellach F, Vilardell M. Hacia el perfil de médico que necesita la comunidad. Med Clin (Barc) 2006; 127: 136-8.
2. Villanueva-Marcos JL, Millán Núñez-Cortés J, BarónMaldonado M, eds. Estándares para la enseñanza de la medicina. Madrid: Agencia Laín Entralgo/Fundación Lilly; 2006.

3. Thomson O'Brien MA, Freemantle N, Oxman AD, Wolf F, Davis DA, Herrin J. Reuniones y talleres de educación continua: efectos sobre la práctica profesional y los resultados de la atención. In: Biblioteca Cochrane Plus, n. ${ }^{03}$, 2008. URL: http://www.biomedexperts.com/ Abstract.bme/11406063/Continuing_education_meetings_and_workshops_effects_on_professional_practice_and_health_care_outcomes. [29.03.2009].

4. Pallie W, Carr DH. The McMaster medical education philosophy in theory, practice and historical perspective. Med Teach 1987; 9: 59-71.

5. Morales-Bueno P, Landa-Fitzgerald V. Aprendizaje basado en problemas. Problem-based learning. Theoría 2004; 13: $145-7$.

6. Alameda-Cuesta A, Alcolea-Cosín MT, Antón-Nardiz $\mathrm{MV}$ et al. El aprendizaje basado en problemas: una herramienta para toda la vida. ABP en la Escuela de Enfermería de la Comunidad de Madrid. Madrid: Agencia Laín Entralgo; 2004.

7. Fernández-Martínez M, García-Sánchez JN, De CasoFuertes A, Fidalgo-Redondo R, Arias-Gundín O. El aprendizaje basado en problemas. Revisión de estudios empíricos internacionales. Revista de Educación 2006; 341: 397-418.

8. Salinas-Sánchez AS, Hernández-Millán I, Virseda-Rodríguez JA, et al. El aprendizaje basado en problemas en la enseñanza de la urología. Modelo de la Facultad de Medicina de la Universidad de Castilla-La Mancha. Actas Urol Esp 2005; 29: 8-15.

9. Neville AJ, Norman GR. PBL in the Undergraduate MD Program at McMaster University: three iterations in three decades. Acad Med 2007; 82: 370-4.

10. Spencer JA, Jordan RK. Learner centred approaches in medical education. BMJ 1999; 318: 1280-3.

11. Koh GC, Khoo HE, Wong ML, Koh D. The effects of problem-based learning during medical school on physician competency: a systematic review. CMAJ 2008; 178: 34-41.

12. Schmidt HG, Vermeulen L, Van der Molen HT. Long-term effects of problem-based learning: a comparison of competencies acquired by graduates of a problem-based and a conventional medical school. Med Educ 2006; 40: 562-7.

13. Hoffman K, Hosokawa M, Blake R Jr, Headrick L, Johnson G. Problem-based learning outcomes: ten years of experience at the University of Missouri-Columbia School of Medicine. Acad Med 2006; 81: 617-25.

14. Blake RL, Hosokawa MC, Riley SL. Student performances on step 1 and step 2 of the United States Medical Licensing Examination following implementation of a 
problem-based learning curriculum. Acad Med 2000;

75: 66-70.

15. Enarson C, Cariaga-Lo L. Influence of curriculum type on student performance in the United States Medical Licensing Examination Step 1 and Step 2 exams: problembased learning vs. lecture-based curriculum. Med Educ 2001; 35: 1050-5.

16. Nandi PL, Chan JN, Chan CP, Chan P, Chan LP. Undergraduate medical education: comparison of problembased learning and conventional teaching. Hong Kong Med J 2000; 6: 301-6

17. Neville AJ. Problem-based learning and medical education forty years on. A review of its effects on knowledge and clinical performance. Med Princ Pract 2009; 18: 1-9.

18. Ministerio de Educación y Ciencia. Orden ECI/332/2008, de 13 de febrero (Ministerio de Educación y Ciencia), por la que se establecen los requisitos para la verificación de los títulos universitarios oficiales que habiliten para el ejercicio de la profesión de médico. BOE n. ${ }^{\circ} 40,15$ de febrero de 2008. p. 8351-5.

19. Communiqué of the Conference of Ministers responsible for Higher Education in Berlin on 19 September 2003. 'Realising the European Higher Education Area' URL: http://www.sedem.org/Comunicado\%20de\%20 Berlin\%202003.pdf. [20.01.2009].

20. Towards the European Higher Education Area. Commu- niqué of the meeting of European Ministers in charge of Higher Education in Prague on May 19th 2001. URL: http://www.sedem.org/Comunicado\%20de\%20Praga-2001.pdf. [20.01.2009].

21. The Bologna Declaration of 19 June 1999. Joint declaration of the European Ministers of Education. URL: http:// www.sedem.org/bologna_declaration1.pdf. [20.01.2009].

22. World Federation for Medical Education. Association for Medical Education in Europe. Statement on the Bologna process and medical education. URL: http://www. sedem.org/bolognadeclaration\%20.htm. [30.01.2009].

23. Haynes RB. Of studies, syntheses, synopses, summaries, and systems: the ' $5 \mathrm{~S}$ ' evolution of information services for evidence-based healthcare. Evid Based Med 2006; 11: 162-4.

24. Sackett DL, Rosenberg, WM, Gray JAM, Haynes RB, Richardson WS. Evidence based medicine: what is and what it isn't. BMJ 1996; 312: 71-2.

25. Álvarez-Montero S., Caballero-Martínez MA, GallegoCasado P, et al. Sesiones basadas en escenarios clínicos: una aplicación del aprendizaje basado en la resolución de problemas. Medifam 2001; 11: 83-91.

26. Torio-Durántez J, García-Tirado MC. El aprendizaje basado en problemas. Tribuna Docente 2002; 3: 65-71, 77-84.

27. Snadden D, Thomas M. The use of portfolio learning in medical education. Med Teach 1998; 20: 192-9.

\section{Anexo 1. Situación clínica para el ABRP (caso clínico para trabajo en tres sesiones tutorizadas)}

\section{Primera sesión tutorizada}

Acude a consulta un varón de 20 años solicitando una receta de Antabús ${ }^{\circledR}$ para su padre: han venido a vivir hace poco a esta zona y el padre no puede acudir a la consulta. Interrogado, el joven informa sobre los problemas de su padre con el alcohol y de que tiene 'algo de azúcar en la sangre'.

Dos años después el paciente consulta por dolores en masa muscular de nalgas, que aparecen al caminar, y dificultad para mantener relaciones sexuales completas por imposibilidad de mantener una erección eficaz, desde hace meses. Admite, desde al menos un año, dolor en las pantorrillas cuando camina rápido o cuesta arriba, lo que le obliga a detenerse. Tiene 50 años y entre sus antecedentes destaca: fumador de 20-30 cigarrillos al día desde los 23 años y bebedor excesivo desde hace unos 15 y hasta hace un año, que dejó porqué sufrió una pancreatitis aguda. Cumple criterios de bronquitis crónica simple y nunca se ha planteado dejar de fumar. Fue diagnosticado de hiperglucemia hace ocho años, pero admite no haber tomado al respecto ninguna precaución dietética ni de cambio de estilo de vida. Tampoco ha realizado los controles que sus médicos previos le propusieron. Es dueño de una empresa de publicidad. Está divorciado y vive con sus dos hijos de 20 y 19 años. No hace ejercicio y su trabajo, además de ser sedentario, le obliga a comer con frecuencia con clientes y proveedores en restaurantes. Pasa la mayor parte del día trabajando y cuando vuelve a su casa no le apetece cocinar nada, por lo que cena cualquier cosa: cereales, leche con cacao....

Entre sus antecedentes familiares destaca la presencia de varios diabéticos tipo 2 no insulinodependientes en la familia paterna. Su madre 
es hipertensa, sin complicaciones. No hay antecedentes de enfermedad cardiovascular ni de tumores. En la exploración física destacan como datos relevantes obesidad con IMC de $35 \mathrm{~kg} / \mathrm{m}^{2}$, hipertrofia parotídea bilateral, TA de 160/100 $\mathrm{mmHg}$ y glucosa capilar de $160 \mathrm{mg} / \mathrm{dL}$ dos horas después del desayuno. El estudio clínico y analítico realizado ponen de manifiesto una glucemia basal sangre de 160 y hemoglobina glucosilada de $10,5 \%$, colesterol total 300, HDL 30, LDL 200, triglicéridos 450, GOT 56, GPT 60, GGT 90, microalbuminuria $>250 \mathrm{mg} /$ día, fondo de ojo sin lesiones sugerentes de retinopatía diabética y ECG normal.

\section{Segunda sesión tutorizada}

Han pasado cinco años desde la primera visita. El control de los problemas del paciente es bueno excepto porque no ha sido capaz de dejar de fumar. Refiere que desde hace meses está triste y desganado: nada le apetece ni le emociona. Ha perdido apetito y peso, tiene ganas de llorar y de mandar todo a paseo. Manifiesta dificultades para conciliar y mantener el sueño. Piensa en la muerte como una escapatoria, aunque no tiene ideación autolítica. Admite tener problemas en su empresa debido a que otras, de las que es proveedora, recortan gastos en publicidad: la empresa no marcha bien y los hijos no colaboran en nada. Dice que sólo piensan en gastar y nada en trabajar. A veces le entran ganas de 'mandar todo a paseo y que les zurzan a los hijos y a la empresa' (sic). Y para colmo se ha venido a vivir con él y sus hijos su exmujer que estaba fuera de España y está buscando trabajo.

\section{Tercera sesión tutorizada}

Han pasado 10 años desde que se conocieron médico y paciente. Desde aquella primera consulta el paciente ha seguido revisiones clínicas y analíticas con regularidad y el control de sus factores de riesgo cardiovascular es bueno. Incluso ha conseguido dejar de fumar.

Comenta en una consulta rutinaria que desde hace meses tose más por las mañanas y ocasionalmente con sangre. Le preocupa que pueda ser algo malo. La radiografía simple de tórax muestra una imagen cavitada en LSI y un aumento de densidad en LMD y LID. Las biopsias fueron diagnosticas de Adenocarcinoma de pulmón bilateral. El TAC toracoabdominal confirmó los hallazgos anteriores junto con una posible afectación de ambas suprarrenales. Ha sido tratado quirúrgicamente de la lesión en pulmón izquierdo y sometido a quimioterapia (dos sesiones) para el tratamiento de la lesión en pulmón derecho. La última sesión fue muy mal tolerada. Le han dado de alta en el hospital con oxigenoterapia domiciliaria, Tramadol retard $200 \mathrm{mg} / 12 \mathrm{~h}$ por vía oral, omeprazol $20 \mathrm{mg} / 24 \mathrm{~h}$ por vía oral, y su tratamiento habitual.

Vas a verle a su casa y te dice que ya está harto y que en el hospital le han comentado que si se encuentra con ganas y fuerte le darían otra sesión de quimioterapia aunque queda a criterio del paciente. Ha comenzado a leer revistas sobre medicinas alternativas y se está hinchando a vitaminas y productos de herbolario. Está muy delgado y tiene poco apetito además de ocasionales dificultades para tragar. Refiere dolor torácico en costado izquierdo, de características pleuríticas, que ha aumentado desde el alta y disnea de moderadospequeños esfuerzos, estable. No duerme bien, aunque refiere estar bien de ánimo; incluso hay momentos en que no se puede estar quieto y no puede parar de hacer cosas. El paciente conoce el diagnostico perfectamente, dice que confía en ti y que ya está harto de médicos y de medicinas y que no quiere saber más del hospital ni de la quimioterapia, porque la última vez casi no lo cuenta (sic). No quiere que sus hijos tengan mucha información sobre su enfermedad. Te pregunta tu opinión, y sobre todo se plantea dejar de ir a las sesiones de quimioterapia, estar en su casa hasta que llegue el momento y 'que salga el sol por donde sea'. 
Anexo 2. Metodología y objetivos de aprendizaje definidos para la situación clínica presentada.

\section{Primera sesión tutorizada}

\section{Metodología}

- Presentación del grupo.

- Elección, por parte de los alumnos, del director y secretario de la sesión entre ellos. El cargo se irá rotando en cada sesión para que todos asuman alguna de las responsabilidades adjuntas.

- El director comienza la lectura del caso, anima y coordina las intervenciones y controla el cumplimiento de objetivos en el tiempo disponible.

- Los alumnos discuten el caso en grupo, identifican los problemas del paciente y sus carencias para responderlos, eligen de entre sus dudas aquellas sobre las que van a trabajar y responder individualmente.

- El secretario toma nota escrita de la evolución de la sesión, ordena las ideas y expone a todos su resumen al final, donde se recogen las tareaspreguntas a resolver para la siguiente sesión.

\section{Objetivos}

- Detectar y resolver el problema ético planteado en la primera consulta del hijo.

- Problemas clínicos

Problema agudo: utilizando el razonamiento clínico llegar del síntoma al diagnóstico de Claudicación intermitente. Revisar su tratamiento.

Problemas crónicos: a) Riesgo cardiovascular: actualizar el diagnóstico, abordaje y seguimiento de tabaquismo, hipertensión arterial, diabetes mellitus, hiperlipemia, obesidad. b) Control del paciente con pluripatología y polifarmacia: proponer el mejor tratamiento para este paciente.

- Contexto socio-familiar: su influencia en el paciente; posibles intervenciones.

\section{Segunda sesión tutorizada}

\section{Metodología}

- Elección entre los alumnos de director y secretario de la sesión.

- El director comienza resumiendo el caso clínico, enumera las preguntas elegidas para res- ponder durante la primera sesión e invita a cada uno de sus compañeros a aportar y argumentar sus respuestas.

- Terminada la fase anterior, el director continúa la lectura del caso clínico, anima/coordina las intervenciones de sus compañeros y controla cumplimiento de objetivos en tiempo previsto.

- Los alumnos discuten el caso en grupo, identifican los problemas del paciente y sus carencias para responderlos, eligen de entre sus dudas aquellas sobre las que van a trabajar y responder individualmente.

- El secretario toma nota escrita de la evolución de la sesión, ordena las ideas y expone a todos su resumen al final, donde se recogen las tareaspreguntas a resolver para la siguiente sesión.

\section{Objetivos}

- Escucha activa: revisamos y discutimos las respuestas propuestas por cada alumno.

- Búsquedas bibliográficas: revisamos las que aportan los estudiantes y evaluamos su calidad.

- Problema clínico: utilizando el razonamiento clínico, llegar del síntoma al diagnóstico de Depresión mayor; revisar su tratamiento.

\section{Tercera sesión tutorizada}

\section{Metodología}

- Elección entre los alumnos de director y secretario de la sesión.

- El director comienza resumiendo el caso clínico, enumera las preguntas elegidas para responder durante la primera sesión e invita a cada uno de sus compañeros a aportar y argumentar sus respuestas.

- Terminada la fase anterior, el director continúa la lectura del caso clínico, anima/coordina las intervenciones de sus compañeros y controla el cumplimiento de objetivos en tiempo previsto.

- Los alumnos discuten el caso en grupo, identifican los problemas del paciente y sus carencias para responderlos, eligen de entre sus dudas aquellas sobre las que van a trabajar y responder individualmente. Estas respuestas serán plasmadas por escrito, sin discusión de grupo, y entregadas al tutor-facilitador. 
- El secretario toma nota de la evolución de la sesión y expone a todos su resumen al final, donde se recogen las tareas-preguntas a resolver individualmente y que entregarán resueltas al tutor.

\section{Objetivos}

- Escucha activa: revisamos y discutimos las respuestas propuestas por cada alumno.
- Búsquedas bibliográficas: revisamos las que aportan los estudiantes y evaluamos su calidad.

- Problema clínico:

Decidir si estamos ante un enfermo terminal. Abordaje integral del enfermo terminal: a) Respeto y autonomía del paciente; b) Tratamiento sintomático: dolor, disnea, problemas de alimentación, problemas psicológicos; c) Dinámica familiar. El cuidado de los cuidadores; d) Relación entre niveles asistenciales. 